\title{
A GLIMPSE INTO CORONAVIRUS DISEASE-19 SCENARIO
}

\section{Introduction}

Coronavirus disease-19 (COVID-19) caused by SARS-COV-2 was first recognized in Wuhan, China in December 2019. It is a beta virus closely linked to the SARS virus. It is believed to have originated in bats, subsequently spreading to cats, and then to humans in China.

\section{Transmission}

Transmission occurs from the symptomatic people to others by close contact through respiratory droplet. The other mode of transmission is by direct contact through contaminated objects and surfaces. The highest shedding of the virus is from upper respiratory tract. The incubation period is up to 14 days. But during the presymptomatic period, i.e. 1-3 days before the onset of symptoms, patients can transmit the virus.

\section{Clinical Profile}

Forty percent of cases are mild while $40 \%$ are moderate cases and only $15 \%$ develop severe disease which requires hospitalization and oxygen support. Five percent develop the critical disease with complications such as respiratory failure, ARDS, sepsis, septic shock, multiple organ failure including AKI and cardiac disease. Risk factors that increase the severity of the disease and death include older age, smoking, noncommunicable disease, hypertension, cardiac disease, chronic lung disease, etc. Increased morbidity is associated with older age, sequential organ failure assessment (SOFA) score, D-dimer $>1 \mu \mathrm{g} / \mathrm{L}$. The study reveals median viral survival is 20 days and maximum has been reported for up to 37 days.

Symptoms of COVID-19 include flu-like symptoms, e.g. dry cough, fever, dryness of mouth, difficulty in breathing, headaches, body aches along with neurological and mental manifestations including delirium and encephalopathy, agitation, stroke, anosmia, anxiety, depression.

Neurological manifestation without respiratory manifestations has been also reported. Sixty-five percent of patients with COVID-19 in ICU showed signs of confusion and 69\% experienced agitation. A case report of GBS and meningoencephalitis has also been reported. Clinical manifestations are milder in young as compared to adults. Even in infants, the disease is milder.

\section{Laboratory Diagnosis}

World Health Organization recommends laboratory diagnosis of all suspected cases via the collection of upper respiratory tract specimens for testing by RT-PCR or even specimens of the lower respiratory tract. SARS-COV-2 antibody tests are not recommended for the diagnosis of current infections with COVID-19. In hospitalized patients with confirmed COVID-19, repeated URTS and LRTS samples are used to test virus clearance as well.

\section{Prevention and Treatment}

In order to disrupt the transmission of the virus all persons suspected or confirmed should immediately be isolated. All these people must wear a medical mask in the isolation room and should be kept at $1 \mathrm{~m}$ distance from each other. All patients must cover the nose and mouth while coughing and sneezing with a tissue of flexed elbow. Standard precautions should be applicable including hand hygiene, use of PPE, prevention of needlestick injury, safe waste management, cleaning and disinfection of pieces of equipment, and cleaning of the environment. Along with these we must also apply contact and droplet precautions including gloves, medical mask, use of eye protection like googles. While performing aerosol-generating procedures use appropriate PPE and fit tested particulate respirators (N95/equivalent/higher level of protection).

\section{Impact on Economy}

COVID-19 is worsening the global economic health every week. It has brought the world economy to a standstill. Containment measures are leading to a further downward prediction of global GDP growth. In mid April, the International Monetary fund (IMF) stressed that global economy was going to experience its worst recession since the great depression. It revised its projection of growth in the trade of goods and services from an earlier estimated $3 \%$ to a negative $9 \%$. World Trade Organization measures trade in terms of an average change in exports and imports.

Hemant Sharma

Head, Department of Medicine NDMC Medical College and Hindu Rao Hospital Malka Ganj, Delhi 